\title{
The evolutionary history of a mammal species with a highly fragmented range: the phylogeography of the European snow vole
}

\author{
R. Castiglia ${ }^{1}$, F. Annesi ${ }^{1}$, B. Kryštufek ${ }^{2}$, M. G. Filippucci ${ }^{3}$ \& G. Amori ${ }^{4}$ \\ 1 Dipartimento di Biologia Animale e dell'Uomo, Università di Roma 'la Sapienza', Rome, Italy \\ 2 Science and Research Centre of Koper, University of Primorska, Koper, Slovenia \\ 3 Dipartimento di Biologia, Università di Roma 'Tor Vergata', Rome, Italy \\ 4 Institute of Ecosystem Studies, CNR, Rome, Italy
}

\section{Keywords}

Chionomys; cytochrome $b$; fragmented distribution; glacial refugia; mtDNA; phylogeography.

\section{Correspondence} \\ Riccardo Castiglia, Dipartimento di Biologia \\ Animale e dell'Uomo, Università di Roma 'la \\ Sapienza', via A. Borelli 50, 00161 Rome, \\ Italy. Tel: + 003906 49918122; Fax: + 0039 \\ 064457516 \\ Email: castiglia@uniroma1.it \\ Editor: Jean-Nicolas Volff \\ Received 22 January 2009; revised 17 June \\ 2009; accepted 17 June 2009
}

doi:10.1111/j.1469-7998.2009.00612.x

\begin{abstract}
The European snow vole Chionomys nivalis has a patchy distribution restricted to rocky habitats across southern Europe and the Near and Middle East. We carried out a phylogeographic study to provide a biogeographic scenario, based on molecular data, outlining the major processes that determined the current distribution of the species. The samples include 26 snow voles from 14 different populations across the entire species range from Spain to Anatolia and Israel. Nearly complete sequences (1037 bp) of the mitochondrial gene for cytochrome $b$ were sequenced. Relationships among haplotypes were inferred with neighbourjoining, maximum likelihood, maximum parsimony analyses and minimum spanning network. An analysis of mismatch distribution was used to cast light on past demographic expansion. We found 22 different haplotypes that fall into six distinct lineages, all but one is supported by high bootstrap values with all methods. Four lineages are allopatric (Tatra Mts., Iberia, Balkans and Middle East) while divergent haplotypes from two lineages show sympatry in the Alps and the Apennines. The basal relationships of these lineages could not be established by any tree. The mean pairwise genetic distance between lineages ranges from 2.4 to $4.2 \%$. The shape of the mismatch distribution indicated a past expansion event dating back to between 158000 and 84000 years ago. These data can be interpreted with the existence of southern glacial refugia (Iberia, Balkans, Middle East and Italy) and one additional northern glacial refugium. The lack of phylogenetic resolution among lineages and the shape of mismatch distribution are indicative of a simultaneous and rapid splitting due to a relatively fast initial expansion of populations. Moreover, the analysis supports the hypothesis of the European origin of $C$. nivalis and its subsequent eastward dispersion during the Middle Pleistocene.
\end{abstract}

\section{Introduction}

European biota is characterized by a strong imprint caused by climatic fluctuations during the last $2 \mathrm{Myr}$ of the Pleistocene. Populations were repeatedly isolated during glacial peaks in different refugia, leading to various re-colonization patterns when the climate improved (Bilton et al., 1998; Taberlet et al., 1998; Schmitt, 2007). Most of the studies that used mitochondrial DNA (mtDNA) markers have indicated that southern Europe, Asia Minor and the Caucasus acted as glacial refugia for taxa that are now widespread (Hewitt, 2000), although some species also persisted in more northern refugia (Kotlík et al., 2006).

In this paper, we focus on the European snow vole Chionomys nivalis a small arvicoline rodent with a patchy range across southern Europe and the Near and Middle East (Fig. 1). The range of the snow vole is highly fragmented mainly due to the habitat requirements of the species. Despite the fact that $C$. nivalis is often found in mountain environments, the species is not physiologically suited to cold environments (Bienkowski \& Marszalek, 1974) and depends on mesic microenvironments in rocky habitats, more specifically on accumulations of rocks and boulders, on cracks in cliffs, escarpments, cave entrances, shafts and crevices in rocky substrate (Luque-Larena, López \& Gosálbez, 2002) regardless of altitude (Kryštufek \& Kovačić, 1989).

On a morphological level, the species was split into 16 (Kratochvil, 1981) or 18 subspecies (Nadachowski, 1991) a 


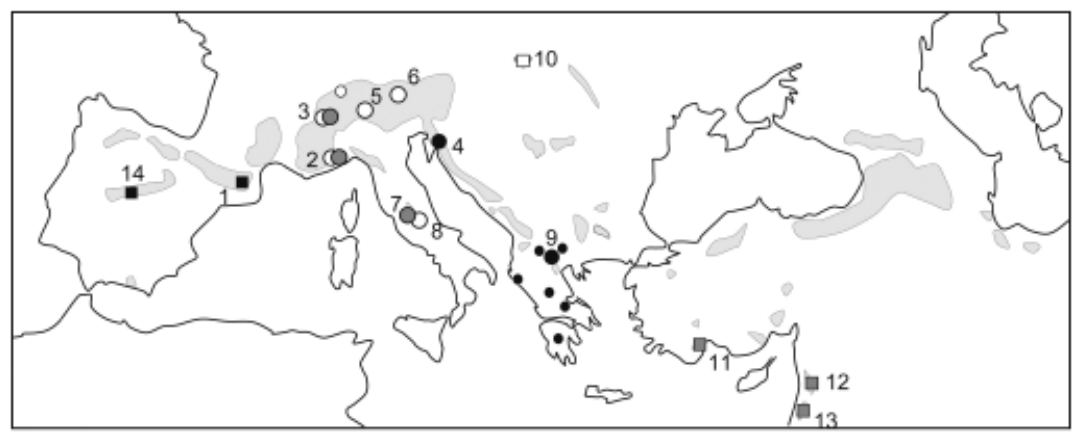

Figure 1 Distribution of Chionomys nivalis (grey areas) and location of the sampled populations. Different symbols and numbers refer to lineages in Figs 2 and 3 and Table 1. Small symbols refer to samples constituted by short sequences from Switzerland and Greece not included in the tree in Fig. 2 (see text for explanation). subdivision that probably reflects local adaptation and which is mainly due to the isolation of populations (Kryštufek, 1999). Allozyme variation of 34 nuclear gene loci and encompassing eight populations from Europe and Asia revealed two independent glacial refugia, the western and the eastern, respectively (Graf, 1982; Filippucci et al., 1991). Moreover, the sample from Israel was so genetically divergent that it has been suggested it should be considered an independent species (Filippucci et al., 1991).

Uncertainty also exists regarding the origin of the species and regarding the biogeographic dynamics that determined the current distribution. In fact, Kryštufek (1999), hypothesized an European origin for C. nivalis and an eastern origin for the other two congeneric species of Chionomys (C. gud and $C$. roberti). Speciation seems to have taken place in the area of the Bosphorus land bridge where land connection between Europe and Asia was frequently disrupted by the Pleistocene sea level oscillations. However, paleontological data fail to provide conclusive evidence supporting the European origin of $C$. nivalis. In fact, during the Middle Pleistocene, the European snow vole was already widespread in Europe (Kowalski, 2001) and also in Asia Minor (Kryštufek \& Vohralík, 2005).

In this context, molecular markers can provide information about the biogeographic history of this species and can also assess the relationships among isolated populations. For this reason, we analysed the phylogeographic relationships among cytochrome $b$ (cyt $b$ ) haplotypes from specimens originating from the entire $C$. nivalis range. The cyt $b$ marker has been chosen because the availability of cyt $b$ sequences of Chionomys in GenBank and for the high substitution rate observed on mammalian mitochondrial genome, which allows to describe intra-specific evolutionary histories (Avise, 2000). More specifically, this study poses the following questions: (1) Did the species originate in Europe or in the Middle East? (2) Was the dispersion a rapid or a gradual process? (3) Did the species follow the refugial model or the distribution was continuous during the glacial periods? (4) Is the European snow vole a monospecific taxon or does it involve cryptic species diversity?

\section{Materials and methods}

In this study, 20 specimens of $C$. nivalis from nine localities were analysed (Fig. 1, Table 1). Furthermore, six sequences of snow voles from five localities (AY513845-9) were downloaded from the GenBank (Jaarola et al., 2004; Galewski et al., 2006).

Total genomic DNAs were obtained from muscle preserved in $80 \%$ ethanol. DNA extraction was performed using the Qiagen method (DNeasy tissue kit, Qiagen, Hilden, Germany). Mitochondrial sequences were isolated combining three different pairs of primers: a long fragment encompassing the entire cyt $b$ was isolated with primers L14723 (5'-ACCAATGACATGAAAAATCATCGTT-3') and H15915 (5'-TCTCCATTTCTGGTTTACAAGAC-3') (Irwin, Kocher \& Wilson, 1991). Two shorter overlapping fragments were also isolated combining primer L14723 with H15553 (5'-TAGGCAAATAGGAAATATCATTCT GGT-3') (fragment named cyt $b 1$ ); and primer L15408 (5'ATAGACAAACTACCATTCCA-3') with H15915 (fragment named cyt $b 2$ ). Amplifications were performed by polymerase chain reaction (PCR) using the following procedure: a first cycle of initial denaturation at $94^{\circ} \mathrm{C}$ for $2 \mathrm{~min}$ followed by 35 cycles with denaturation at $94^{\circ} \mathrm{C}$ for $1 \mathrm{~min}$ annealing temperature $\left(52^{\circ} \mathrm{C}\right.$ for the long fragment, $55^{\circ}$ for cyt $b 1,50^{\circ} \mathrm{C}$ for cyt $b 2$ ) for $1 \mathrm{~min}$ and extension at $72^{\circ} \mathrm{C}$ for $1 \mathrm{~min} 30 \mathrm{~s}$. These steps were followed by extension at $72{ }^{\circ} \mathrm{C}$ for $10 \mathrm{~min}$. Each PCR reaction used 50-500 ng of template DNA, $200 \mathrm{ng}$ of each primer, $0.2 \mathrm{mM}$ of each dNTP and $2 \mathrm{U}$ of Taq polymerase (Promega, Madison, WI) in a final volume of $50 \mu \mathrm{L}$. Amplified products were purified using the Qiagen QIAquick purification kit and prepared for automated sequencing with same primers used for amplification. A fragment of $1040 \mathrm{bp}$ was successfully sequenced from all the samples and used for phylogenetic analysis together with the haplotypes downloaded from the GenBank (Table 1). Nucleotide $(\pi)$ and haplotype diversity together with a minimum spanning network MSN among haplotypes was calculated with Arlequin ver. 3.1 (Excoffier, Laval \& Schneider, 2005).

The appropriate model of substitution was chosen using the Model Test 3.7 program (Posada \& Crandall, 1998). Models of evolution, which provide the best approximation of the data, were chosen for subsequent analysis according to the Akaike information criterion (AIC) and the hierarchical likelihood ratio tests (hLRT). Two different models were selected with the following two criteria: the hLRT identified the Hasegawa, Kishino, Yano (HKY) model (Hasegawa, Kishino \& Yano, 1985) with rate variation 
Table 1 Haplotype name, localities and lineages from the sample of Chionomys nivalis

\begin{tabular}{|c|c|c|c|c|}
\hline $\begin{array}{l}\text { Haplotype (number } \\
\text { of specimens) }\end{array}$ & Locality & Lineage & Site on map & $\begin{array}{l}\text { GenBank accession } \\
\text { numbers }\end{array}$ \\
\hline chio1 (1) & Ciglikara (Turkey) & L1 & 11 & GQ150786 \\
\hline chio2 (1) & Ciglikara (Turkey) & L1 & 11 & GQ150787 \\
\hline chio3 (1) & Ciglikara (Turkey) & L1 & 11 & GQ150788 \\
\hline chio7 (4) & Mt. Pelister (Macedonia) & L2 & 9 & GQ150791 \\
\hline chio8 (1) & Mt. Sneznik (Slovenia) & L2 & 4 & GQ150792 \\
\hline chio9 (1) & Mt. Sneznik (Slovenia) & L2 & 4 & GQ150793 \\
\hline chio5 (1) & Mt. Hermon (Israel) & L1 & 13 & GQ150789 \\
\hline chio6 (1) & Mt. Hermon (Israel) & L1 & 13 & GQ150790 \\
\hline chio19 (1) & Marta Alpi Liguri (Italy) & L6 & 2 & GQ150800 \\
\hline chio11 (1) & Marta Alpi Liguri (Italy) & $\llcorner 4$ & 2 & GQ150794 \\
\hline chio12 (1) & Val Masino (Italy) & $\llcorner 4$ & 5 & GQ150795 \\
\hline chio14 (1) & Val Masino (Italy) & L4 & 5 & GQ150796 \\
\hline chio15 (1) & Valle d'Aosta (Italy) & $\llcorner 4$ & 3 & GQ150797 \\
\hline chio16 (1) & Valle d'Aosta (Italy) & $\llcorner 4$ & 3 & GQ150798 \\
\hline chio20 (1) & Valle d'Aosta (Italy) & L6 & 3 & GQ150801 \\
\hline chio17 (1) & Gran Sasso (Italy) & L4 & 7 & GQ150799 \\
\hline chio21 (1) & Duchessa (Italy) & L6 & 8 & GQ150802 \\
\hline chio13 (2) & Trento (Italy) & $\llcorner 4$ & 6 & AY513845 \\
\hline chio10 (1) & $\begin{array}{l}\text { Prve Rohačske Pleso Lake, } \\
\text { West Tatra Mts. (Slovakia) }\end{array}$ & L3 & 10 & AY513847 \\
\hline chio18 (1) & Girona (Spain) & L5 & 1 & AY513848 \\
\hline chio4 (1) & Saleh, As Suwayda (Syria) & L1 & 12 & AY513849 \\
\hline chio22(1) & Sierra de Gredos (Spain) & L5 & 14 & AM392367 \\
\hline
\end{tabular}

among sites $(+G)$, a proportion of invariable sites $I=0.5875$ and a gamma distribution shape parameter of 0.7221; the AIC selected the transitional model including invariable sites $I=0.6452$ and a gamma distribution shape parameter of 1.0412. The models and parameters were used for neighbour-joining (NJ) and maximum likelihood (ML) trees in PAUP 4.0b10 (Swofford, 1998). For maximum parsimony (MP), we tested different outgroups by equally weighted parsimony. The higher bootstrap values were obtained using multiple outgroups; hence, the sequences from C. roberti (AY513850), Myodes rutilus (AF272638), Microtus arvalis (AY220787) and Microtus oregoni (AF163903) were used as outgroups. MP trees were obtained with PAUP using a heuristic search and tree-bisection-reconnection and random addition of sequences. The robustness of the nodes was assessed by bootstrap with 1000 replicates for MP and NJ and 100 replicates for ML.

Analyses were also repeated using a dataset including short sequences downloaded from the GenBank from six localities of Balkans (Mt. Pirin, Mt. Sar Planina, Velouxi, Xelmos, Tzoumerka and Korakas) and one locality from Switzerland (Pilatus) (accession numbers DQ329150-8 and AY332715-6) (Fig. 1). Because the sequences in the resulting dataset were short $(340 \mathrm{bp})$, this analysis was only used to assign unambiguously these sequences to the lineages identified with the $1073 \mathrm{bp}$ dataset.

To examine the prevalence of multiple hits, saturation plots were evaluated for each substitution type (transitions and transversions) at each codon position. For each pair of haplotypes, the substitution divergence was plotted against total uncorrected sequence divergence $(p)$. The Kimura 2 parameter (K2P) distance between haplotypes was calculated with MEGA 4 (Tamura et al., 2007). Rate homogeneity for nucleotide substitutions among lineages was tested using a relative rate test (Tajima, 1993) with MEGA 4. Each haplotype was compared with the others using the sequence of $C$. roberti as an outgroup. The standard rate $2 \% \mathrm{Myr}^{-1}$ (Wilson et al., 1985) for homeotherms is not applicable for rodents because they have a faster rate (Martin \& Palumbi, 1993).

For the closely related genus Microtus, the rate is very variable varying from $7.5 \%$ (Galbreath \& Cook, 2004) to 6-10\% (Jaarola \& Searle, 2004) and 13.3\% (Conroy \& Cook, 2000).

For the genus Chionomys the estimated range of occurrence of the different species is rather broad and therefore the times of the splits are not well established. Considering the split between Microtus and Chionomys occurred about 1 Mya (Chaline \& Graf, 1988) and that C. nivalis and $C$. roberti are known as separate since Middle Pleistocene (0.78-0.13 Mya) (Nadachowski \& Baryshnikov, 1991), we can roughly estimate the split between $C$. nivalis and $C$. roberti occurring during 1 and 0.78 Mya. Considering the genetic divergence between $C$. nivalis and $C$. roberti $(11.2 \%)$ the rate results comprised between 11.2 and $14.3 \%$. These values corresponds to the upper limit of the range calculated with the calibration obtained for the other Arvicolinae (6-13.3\%) and can be safely used to estimated a range of divergence times in Chionomys.

We analysed the shape of the distribution of pairwise genetic differences between individual haplotypes (mismatch 
distribution) in order to looking for eventual genetic signatures indicating a rapid expansion of populations (and possibly to infer the time when the expansion began). The mismatch distribution is affected by historical demographic changes of populations: population expansion can generate a unimodal distribution, stable populations typically produce a multimodal distribution and a sudden reduction in population size initially generates an L-shaped distribution (Slatkin \& Hudson, 1991; Rogers \& Harpending, 1992).

In the model of Rogers \& Harpending (1992), a population in equilibrium with an initial size of $N_{0}(N$ being the effective female population size) suddenly changes to $N_{1}$ at $\tau$ units of mutational time. This process generates a mismatch distribution described approximately by a Poisson distribution. The parameters of the model are described by: $\theta_{0}=2 N_{0} u ; \theta_{1}=2 N_{1} u$ with $\tau=2 u t$ where $u$ is the sequence's mutation rate per generation. The significance of the $(\tau)$ difference between the expected and observed mismatch distribution was tested following the parametric bootstrap approach (Schneider \& Excoffier, 1999).

\section{Results}

Twenty-two haplotypes, named chio1-chio22 (1073 bp), were identified among sequences from the total dataset. The two haplotypes from the Alps (Trento) downloaded from the GenBank were found to be identical (chio13). The number of polymorphic sites was 119 with 128 substitutions (117 transitions and 11 transversions). Haplotype diversity was high being $0.98 \pm 0.021$ and the overall nucleotide diversity is $0.027 \pm 0.013$. The base composition of cyt $b$ (C $28.2-29.2 \%$, mean 28.6; T 27.6-28.2\%, mean 27.9; A $30.7-31.4 \%$, mean 30.9 ; G $12.3-13.0 \%$, mean 12.7 ) and the absence of stop codons and indels are consistent with other Chionomys sequences downloaded from the GenBank (Jaarola et al., 2004) and with other mammalian cyt $b$ sequences (Irwin et al., 1991). The distribution of variation across the codon position (first position $13.7 \%$ considering all variable sites, second $5.1 \%$, third $81.2 \%$ ) was as expected for genuine functional cyt $b$ sequences (Triant \& Dewoody, 2007). Moreover, the distribution of the variable amino acid sites fits the structural model of variable and conserved regions on cyt $b$ (Irwin et al., 1991).

Saturation plots indicate that there is no evidence of saturation in any codon position. All three codon positions show an almost linear increase of transitions and transversions across the range of sequence divergence examined.

All the trees we generated with the different methodologies (MP, NJ and ML) gave similar topologies, so only the ML tree is shown in Fig. 2. The monophyly of $C$. nivalis is well supported by bootstrap values (99-100\%). The $22 C$. nivalis haplotypes fall into six distinct lineages (L1-L6). These are all supported by high bootstrap values with all the methods except L6 lineage which is supported only by MP (61\%). However, haplotypes of L6 lineage cluster together also in NJ and ML trees. The L3 lineage is constituted by one single specimen haplotype (chio10). The distribution of the six lineages shows a clear geographical pattern. Lineage L1 includes haplotypes from the Middle East including Syria, Israel and Turkey. The L4 and L6 lineages are present in the Alps and also include haplotypes from two localities in the Apennines. The L2 lineage contains two distant populations from the Balkans. The L5 lineage is found in Spain. In addition, the divergent haplotype forming lineage L3 (chio10) belongs to Slovakia.

When we include in the phylogenetic analysis the shorter sequences from Greece, they unambiguously are assigned to the Balkan lineage (L2). In the same way the two sequences from Switzerland fall in the Alpine L4 lineage (Fig. 1).

The basal relationships of these lineages are not resolved by any tree. A minimum spanning network shown in Fig. 3 illustrates the relationships among haplotypes and the number of substitutions dividing them. A limited number of substitutions (1-18) separate haplotypes within each lineage, while a higher number (20-30) separates the different lineages. The only exception is constituted by lineages L6 and L4 which are separated by 15 substitutions only.

The mean pairwise genetic distance (K2P) between lineages ranges between 2.4 and $4.2 \%$.

The shape of the mismatch distribution for the pooled haplotypes was unimodal (not shown). We observed a nonsignificant $P$ value for the mismatch distribution test of goodness of fit (Harpending's Raggedness index $=0.009$, $P=0.34$ ). Therefore, we inferred a scenario of expansion of population in the past. The time since the start of expansion is indicated by the modal value $(\tau)$, which is 33.99 (95\% CI 25.88-38.05). The relative rate test did not reject the molecular clock hypothesis. Considering the molecular clock we applied, we estimated that the time of expansion occurred in a time that ranges between 158000 and 84000 years ago.

\section{Discussion}

\section{Distribution of the mtDNA lineages}

The snow vole, shows an unusual distribution among all the European mammals which, is wide and fragmented (Mitchell-Jones et al., 1999). The samples used in this analysis cover almost all this wide distribution. For this reason, through this present phylogeographic analysis, it is possible to track the major events characterizing the evolutionary history of populations. The phylogenetic trees obtained from the 22 cyt $b$ haplotypes comprise six divergent lineages (Figs 2 and 3). Four of them are allopatric, while divergent haplotypes from two supported lineages (L4 and L6) show broad sympatry in the Alps and the Apennines (Fig. 1). The presence of structured populations, contradicts previous analyses based on allozymes which had revealed the presence of only two separated lineages in Europe (the western and the eastern lineage; Graf, 1982; Filippucci et al., 1991). This discrepancy can easily be explained if one considers the higher resolution of rapidly evolving mtDNA markers compared with allozymes. The distribution of these lineages matches with the known glacial refugia of the European biota. Although we have not detailed enough sampling and these results have to be confirmed with nuclear loci (e.g. 
Figure 2 Maximum likelihood tree. Numbers correspond to the percentage of bootstrap replicates supporting the node (percentage over 1000 replicates for neighbour-joining, percentage over 1000 replicates for equally weighted maximum parsimony, percentage over 100 replicates for maximum likelihood). The six lineages (L1-L6) are also indicated.

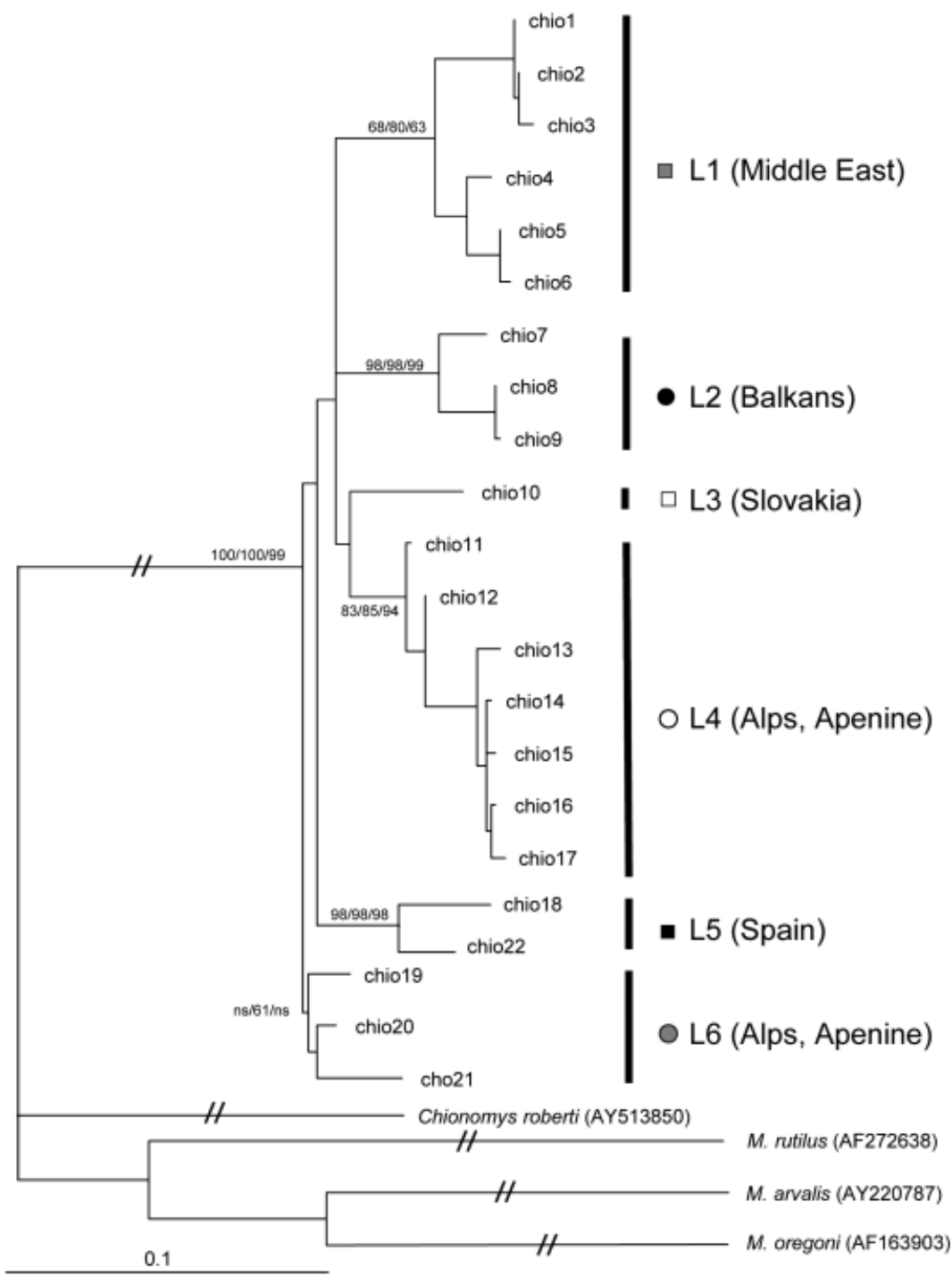

microsatellite loci), we suggest the existence of at least four southern glacial refugia, namely in Iberia, the Balkans, the Middle East and Italy (the Alps and Apennines). Moreover, a single divergent haplotype from Tatra Mts. provide evidence regarding the possible existence of an additional glacial refugium in central Europe, to the north of the main southern refugia in the European peninsulas and the Near East. Several recent studies have confirmed the presence of northern refugia for small temperate mammals contradicting a widespread belief concerning the exclusive existence of southern refugia (e.g. Myodes glareolus; Kotlík et al., 2006). However, the small temperate mammals which survived glacial peaks in northern refugia are usually of wide latitudinal occurrence. In contrast, the European snow vole predominantly inhabits the Mediterranean mountains (Shenbrot \& Krasnov, 2005).

The coexistence of different lineages in the Alps and Apennines can be due to a secondary admixture of divergent populations or, alternatively, it may be due to the incomplete lineage sorting of two old lineages.

Considering the wide range of the European snow vole, which encompasses southern Europe and the Middle East, an important question to be discussed regards the centre of origin of the species. MST topology is potentially informative in localizing the ancestral haplotypes. The two lineages found in the Alps and the Apennines (L4 and L6) hold a central position in the MST network compared with other haplotypes. The central position of these lineages supports the hypothesis concerning the European origin of $C$. nivalis and its subsequent eastward dispersion across the Bosphorus land bridge into Asia (Kryštufek, 1999).

\section{The biogeographic dynamics of $C$. nivalis}

The pattern of dispersion from this ancient refugium can be estimated through the topology of the phylogenetic tree as 


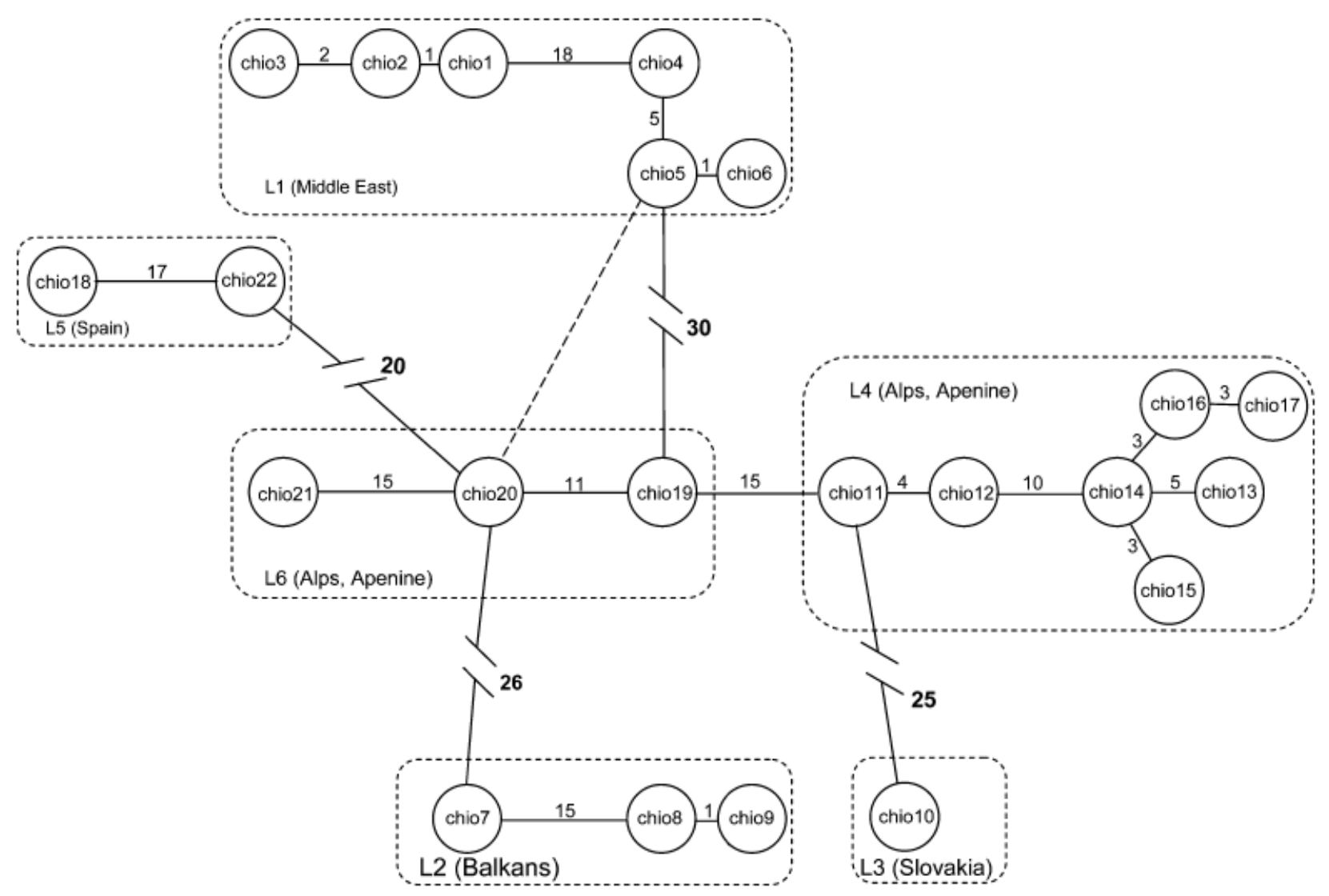

Figure 3 Minimum spanning network of Chionomys nivalis haplotypes. The haplotypes corresponding to the six lineages (L1-L6) identified by the phylogenetic analysis are also indicated.

well as through the analysis of the mismatch distribution. From the tree topology, we have derived two competitive hypotheses. If $C$. nivalis lineages arose from a rapid multiple splitting of a single ancestral population with a subsequent fragmentation into five refugial areas, the relationships among lineages would be unresolved forming a polytomy. Alternatively, if the dispersion took place gradually, during different glacial cycles, some lineages should appear to be more ancient than others and phylogeny should output a series of bifurcations, producing a certain clustering of lineages. Our results clearly support the first hypothesis. For this reason, we hypothesize that the lineages separated from each other through a rather sudden expansion during one single interglacial period, rather than through a gradual shift of species range. The shape of the mismatch distribution also supports this population expansion in the past. Applying the molecular clock, the time of separation calculated both with the slower and the faster clock suggests that the split occurred approximately between 158000 and 84000 years ago. This considerable range of time is due to the uncertainty of the molecular clock calibration. However, the estimated time is much older than the time of Last Glacial Maximum (21000-17000 years ago) and can be placed between at the boundary between Middle and Lower Pleistocene.
The major evolutionary split in the ancestor of the all the extant Chionomys species probably belongs to the first major glaciation in the Early Pleistocene. This event, determined the separation of the ancestor of $C$. gud and C. roberti in the east and $C$. nivalis in the west. A similar explanation has been suggested regarding the separation between Apodemus mystacinus and Apodemus epimelas (Michaux, Bellinvia \& Lymberakis, 2005) (13.3\% divergence in cyt $b$ ). However, while the two eastern species nowadays show a small range localized in the Middle East, a completely different situation arose for $C$. nivalis. During the severe climatic change in the Early Pleistocene, $C$. nivalis was probably restricted to rocky habitats, which are abundant in the Apennine and the Alpine arch. Following this, at a later date during the end of the Middle Pleistocene, the European snow vole displayed a rapid expansion that was probably possible thanks to a mild interglacial period. Subsequent glacial oscillations favoured the evolution of the $C$. nivalis major mtDNA lineages (L1-L6) in separate refugial areas. These lineages are well separated (mean corrected K2P distance: $2.4-4.2 \%$ ), similarly to what was observed in other mammals showing divergent populations in southern refugial areas, for example Apodemus sylvaticus (Michaux et al., 2003), Neomys anomalus (Castiglia et al., 2007), Microtus savii (Castiglia et al., 2008) and Erinaceus europaeus (Seddon 
et al., 2001). Concerning the taxonomic status of the peripheral population from Mt. Hermon, in Israel (locality 13 in Fig. 1) allozymic divergence suggests that this population is a separate species (Filippucci et al., 1991). However, the here presented results of mtDNA analysis do not support such a view. In fact, this population is no older than the others and the calculated genetic distance indicates affinity with other samples from the Middle East (localities 11 and 12). Considering these estimated times of divergence, the proposed scenario fits with fossil evidence. In fact, during the Middle Pleistocene, the European snow vole was widespread in Europe (Kowalski, 2001) and already present in Asia Minor (Emirkaya-2 and the island of Chios) (Kryštufek \& Vohralík, 2005).

\section{Conclusion}

We provide, for the first time, a biogeographic scenario, for the major pattern that determined the current distribution of $C$. nivalis, based on molecular data. However, further analyses of other DNA segments, particularly of nuclear genes, are advisable to confirm the proposed pattern. Our reconstruction suggests that populations were subjected to a rapid expansion event that determined the current wide distribution. However, while a rapid expansion of populations is to be expected for species adapted to contiguous habitats, the scenario is different for a species constrained to a patchy habitat. It is important to underline that this expansion does not seem to have occurred for other species of rock-dwelling mammals which nowadays have very small ranges restricted to the mountains of south Europe and the Near and Middle East. Examples of this include Dinaromys bogdanovi, Dryomys laniger, Crocidura arispa, Crocidura serezkiensis, Crocidura zarudnyi, A. mystacinus and A. epimelas. Slight differences in ecological requirements can account for this observation. Therefore, additional genetic studies on $C$. nivalis populations and other rock-dwelling mammals are needed to better understand the role of habitat heterogeneity on genetic variability at a smaller scale.

\section{Acknowledgement}

This work has been supported by funds 'progetti di ricerca di università' and 'progetti di ricerca di Ateneo federato' (Università di Roma 'La Sapienza'). We thank the three referees for useful comments on earlier version of this paper. Tissues of snow voles from Apennines were gently provided by Paola Tuccinardi and Alessandra Buscemi.

\section{References}

Avise, J.C. (2000). Phylogeography: the history and formation of species. Cambridge, MA: Harvard University Press.

Bienkowski, P. \& Marszalek, U. (1974). Metabolism and energy budget in the snow vole. Acta Theriol. 19, 55-67.

Bilton, D.T., Mirol, P.M., Mascheretti, S., Fredga, K., Zima, J. \& Searle, J.B. (1998). Mediterranean Europe as an area of endemism for small mammals rather than a source for northwards postglacial colonization. Proc. Roy. Soc. Lond. Ser. B: Biol. Sci. 265, 1219-1226.

Castiglia, R., Annesi, F., Aloise, G. \& Amori, G. (2007). Mitochondrial DNA reveals different phylogeographic structures in the water shrews Neomys anomalus and $N$. fodiens (Insectivora: Soricidae) in Europe. J. Zool. Syst. Evol. Res. 45, 255-262.

Castiglia, R., Annesi, F., Aloise, G. \& Amori, G. (2008). Systematics of the Microtus savii complex (Rodentia, Cricetidae) via mitochondrial DNA analyses: paraphyly and pattern of sex chromosome evolution. Mol. Phylogenet. Evol. 46, 1157-1164.

Chaline, J. \& Graf, J.D. (1988). Phylogeny of the Arvicolidae (Rodentia): biochemical and paleontological evidence. J. Mammal. 69, 22-33.

Conroy, C.J. \& Cook, J.A. (2000). Phylogeography of Microtus longicaudus, a post-glacial colonizer. Mol. Ecol. 9, 165-175.

Excoffier, L., Laval, G. \& Schneider, S. (2005). Arlequin ver. 3.0: an integrated software package for population genetics data analysis. Evol. Bioinform. Online 1, 47-50.

Filippucci, M.G., Fadda, V., Kryštufek, B., Simson, S. \& Amori, G. (1991). Allozyme variation and differentiation in Chionomys nivalis (Martins, 1842). Acta Theriol. 36, 47-62.

Galbreath, K. \& Cook, J. (2004). Genetic consequences of Pleistocene glaciations for the tundra vole (Microtus oeconomus) in Beringia. Mol. Ecol. 13, 135-148.

Galewski, T., Tilak, M., Sanchez, S., Chevret, P., Paradis, E. $\&$ Douzery, E. (2006). The evolutionary radiation of arvicolinae rodents (voles and lemmings): relative contribution of nuclear and mitochondrial DNA phylogenies. BMC Evol. Biol. 6, 80.

Graf, J.-D. (1982). Génétique biochimique, zoogéographie et taxonomie des Arvicolidae (Mammalia, Rodentia). Rev. Suisse Zool. 89, 749-787.

Hasegawa, M., Kishino, H. \& Yano, T. (1985). Dating of human-ape splitting by a molecular clock of mitochondrial DNA. J. Mol. Evol. 22, 160-174.

Hewitt, G. (2000). The genetic legacy of the quaternary ice ages. Nature 405, 907-913.

Irwin, D.M., Kocher, T.D. \& Wilson, A.C. (1991). Evolution of the cytochrome $b$ gene of mammals. J. Mol. Evol. 32, 128-144.

Jaarola, M., Martinkova, N., Gunduz, I., Brunhoff, C., Zima, J., Nadachowski, A., Amori, G., Bulatova, N.S., Chondropoulos, B., Fraguedakis-Tsolis, S., Gonzalez-Esteban, J., Jose Lopez-Fuster, M., Kandaurov, A.S., Kefelioglu, H., da Luz Mathias, M., Villate, I. \& Searle, J.B. (2004). Molecular phylogeny of the speciose vole genus Microtus (Arvicolinae, Rodentia) inferred from mitochondrial DNA sequences. Mol. Phylogenet. Evol. 33, 647-663.

Jaarola, M. \& Searle, J.B. (2004). A highly divergent mitochondrial DNA lineage of Microtus agrestis in southern Europe. Heredity 92, 228-234. 
Kotlík, P., Deffontaine, V., Mascheretti, S., Zima, J., Michaux, J.R. \& Searle, J.B. (2006). A northern glacial refugium for bank voles (Clethrionomys glareolus). Proc. Natl. Acad. Sci. USA 103, 14860-14864.

Kowalski, K. (2001). Pleistocene rodents of Europe. Folia Q. 72, 1-389.

Kratochvil, J. (1981). Chionomys nivalis (Arvicolidae, Rodentia). Acta Sci. Nat. Acad. Sci. Bohemoslov. 15, 1-62.

Kryštufek, B. (1999). Snow voles, genus Chionomys, of Turkey. Mammalia 63, 323-339.

Kryštufek, B. \& Kovačić, D. (1989). Vertical distribution of the Snow vole Microtus nivalis (Martins, 1842) in Northwestern Yugoslavia. Mamm. Biol. 54, 153-156.

Kryštufek, B. \& Vohralík, V. (2005). Mammals of Turkey and Cyprus. Rodentia I: Sciuridae, Dipodidae, Gliridae, Arvicolinae. Koper, Slovenia: University of Primorska.

Luque-Larena, J.J., López, P. \& Gosálbez, J. (2002). Microhabitat use by the snow vole Chionomys nivalis in alpine environments reflects rock-dwelling preferences. Can. J. Zool. 80, 36-41.

Martin, A.P. \& Palumbi, S.R. (1993). Body size, metabolic rate, generation time, and the molecular clock. Proc. Natl. Acad. Sci. USA 90, 4087-4091.

Michaux, J., Bellinvia, E. \& Lymberakis, P. (2005). Taxonomy, evolutionary history and biogeography of the broadtoothed field mouse (Apodemus mystacinus) in the eastern Mediterranean area based on mitochondrial and nuclear genes. Biol. J. Linn. Soc. 85, 53-63.

Michaux, J., Magnanou, E., Paradis, E., Nieberding, C. \& Libois, R. (2003). Mitochondrial phylogeography of the woodmice (Apodemus sylvaticus) in the Western Palearctic region. Mol. Ecol. 12, 685-697.

Mitchell-Jones, A.J., Amori, G., Bogdanowicz, P.J.H., Kryštufek, B., Reijnders, P.J.H., Spitzenberger, F., Stubbe, M., Thissen, J.B.M., Vohralik, V. \& Zima, J. (1999). The atlas of European mammals. London: Poyser.

Nadachowski, A. (1991). Systematics, geographic variation, and evolution of snow voles (Chionomys) based on dental characters. Acta Theriol. 36, 1-45.

Nadachowski, A. \& Baryshnikov, G. (1991). Pleistocene snow voles (Chionomys Miller, 1908) (Rodentia, Mammalia) from Northern Caucasus (USSR). Acta Zool. Cracov. 34, 437-451.

Posada, D. \& Crandall, K.A. (1998). Modeltest: testing the model of DNA substitution. Bioinformatics 14, $817-818$.
Rogers, A.R. \& Harpending, H. (1992). Population growth makes waves in the distribution of pairwise genetic differences. Mol. Biol. Evol. 9, 552-569.

Schmitt, T. (2007). Molecular biogeography of Europe: Pleistocene cycles and postglacial trends. Front. Zool. 4, 11.

Schneider, S. \& Excoffier, L. (1999). Estimation of past demographic parameters from the distribution of pairwise differences when the mutation rates vary among sites: application to human mitochondrial DNA. Genetics 152, 1079-1089.

Seddon, J.M., Santucci, F., Reeve, N.J. \& Hewitt, G.M. (2001). DNA footprints of European hedgehogs, Erinaceus europaeus and E. concolor: Pleistocene refugia, postglacial expansion and colonization routes. Mol. Ecol. 10, 2187-2198.

Shenbrot, G.I. \& Krasnov, B.R. (2005). An atlas of the geographic distribution of the Arvicoline rodents of the world (Rodentia, Muridae: Arvicolinae). Sofia: Pensoft Publishers.

Slatkin, M. \& Hudson, R. (1991). Pairwise comparisons of mitochondrial DNA sequences in stable and exponentially growing populations. Genetics 129, 555-562.

Swofford, D.L. (1998). PAUP* 4.0 - phylogenetic analysis using parsimony ( ${ }^{*}$ and other methods). Sunderland: Sinauer Associates.

Taberlet, P., Fumagalli, L., Wust-Saucy, A.-G. \& Cosson, J.-F. (1998). Comparative phylogeography and postglacial colonization routes in Europe. Mol. Ecol. 7, 453-464.

Tajima, F. (1993). Simple methods for testing the molecular evolutionary clock hypothesis. Genetics 135, 599-607.

Tamura, K., Dudley, J., Nei, M. \& Kumar, S. (2007). MEGA4: Molecular Evolutionary Genetics Analysis (MEGA) software version 4.0. Mol. Biol. Evol. 24, 1596-1599.

Triant, D.A. \& Dewoody, J.A. (2007). The occurrence, detection, and avoidance of mitochondrial DNA translocations in mammalian systematics and phylogeography. J. Mammal. 88, 908-920.

Wilson, A.C., Cann, R.L., Carr, S.M., George, M., Gyllensten, U.B., Helm-Bychowski, K.M., Higuchi, R.G., Palumbi, S.R., Prager, E.M., Sage, R.D. \& Stoneking, M. (1985). Mitochondrial DNA and two perspectives on evolutionary genetics. Biol. J. Linn. Soc. 26, $375-400$ 